\title{
Historia de la cooperación política y económica entre Rusia y México a principios del siglo xxi (2000-2019)
}

\section{History of Political and Economic Cooperation between Russia and Mexico at the Beginning of the 21st Century (2000-2019)}

\author{
Ekaterina Kosevich ${ }^{1} *$ (D) 0000-0002-8056-8426
}

\begin{abstract}
${ }^{1}$ Laboratorio Internacional de Estudios del Orden Mundial y el Nuevo Regionalismo, Facultad de Economía Mundial y Relaciones Internacionales, Universidad Nacional de Investigación, Escuela Superior de Economía (HSE University), Moscú, Federación Rusa.

* Correspondencia: ekaterina.kosevich@gmail.com
\end{abstract}

Resumen. Este artículo analiza las diferentes etapas de las relaciones ruso-mexicanas a finales del siglo xx y principios del xxı, considerando las direcciones principales de desarrollo del comercio entre ambos países, así como los logros esenciales de la cooperación bilateral en las esferas de aviación, energía, transporte, cultura y educación. La autora concluye que, incluso teniendo una serie de puntos en común sobre asuntos internacionales clave, México y Rusia aún no han logrado formar un modelo óptimo y productivo de sus relaciones bilaterales. El estudio tiene claras implicaciones prácticas; en cuanto basado en métodos empíricos de cognición, se formulan recomendaciones para el desarrollo futuro de la cooperación bilateral.

Palabras clave: diplomacia; cooperación internacional; política; economía; comercio.

Abstract. The article is devoted to the study of the evolution of relations between Russia and Mexico at the end of the 20th century and the beginning of the 21 st century. The article discusses the main stages of development of Russian-Mexican cooperation in the diplomatic, economic, cultural and political spheres. The

CÓMO CITAR: Kosevich, E. (2021). Historia de la cooperación política y económica entre Rusia y México a principios del siglo XXI (2000-2019). América Latina en la Historia Económica, 28(3), 1-21. DOI: 10.18232/alhe.1176 
author concludes that even having a number of common ground on key international issues, Mexico and Russia have so far failed to form an optimal and productive model of their bilateral relations. Present study has scientific and practical significance, given the fact that by applying of empirical methods of cognition recommendations for the further development of bilateral cooperation have been formulated.

Key words: diplomacy; international cooperation; politics; economics; trade.

JEL: N10; N46; N76; N96

Recibido: 14 de febrero de 2020.

Aceptado: 05 de octubre de 2020.

Publicado: 23 de abril de 2021.

Organismo patrocinador: Se agradece el apoyo del Programa de Investigación Individual de la Escuela de Economía Mundial y Asuntos Internacionales de la Universidad Nacional de Investigación Escuela Superior de Economía.

Support from the Individual Research Program of the School of World Economy and International Affairs at National Research University - Higher School of Economics is gratefully acknowledged.

Agradecimiento: Me gustaría agradecer a Alejandro Vallina Rodríguez (Departamento de Geografía, Universidad Autónoma de Madrid), por sus útiles comentarios sobre la primera versión de este artículo.

\section{INTRODUCGIÓN}

La temática de las relaciones modernas entre México y Rusia se ha estudiado muy poco, tanto en Rusia como en México. Una parte importante de las publicaciones contemporáneas dedicadas a este tema es de naturaleza circunstancial y se centra en temas del "regreso de Rusia a América Latina”, el problema de implantación de los intereses geopolíticos de Rusia en la esfera de influencia natural de Estados Unidos, así como la "rivalidad” entre Rusia y China en la región.

Como premisa inicial, es necesario acotar que, en las esferas de conocimiento de Rusia, el interés hacia México se desarrolló durante el periodo soviético. Esa primera afirmación está detrás del hecho que ha supuesto que los temas de la evolución del sistema de relaciones internacionales que se ha establecido en el hemisferio occidental y del cambio de equilibrio de poder entre su centro, Estados Unidos y su periferia, América Latina, hayan sido los más desarrollados por los investigadores rusos (Latin America, 2009; USA, 2004). Adicionalmente, las peculiaridades de la inclusión de la región latinoamericana en los procesos globales han formado otro aspecto clave para la ciencia rusa (Lavut, 2012; Sheremetyev y Lapshev 1990).

Cabe señalar que el estudio exhaustivo de México en la ciencia rusa se desarrolló durante mucho tiempo en el marco de la tradición marxista (Shulgovsky, 1967), aunque la desintegración de la Unión Soviética que condujo a la crisis de la metodología soviética exigió que los estudios latinoamericanos desarrollasen un nuevo modelo metodológico; es decir, ir más allá del marco de las actitudes ideológicas cuando se consideran los procesos económicos y sociohistóricos en América Latina y, en particular, en México, inspirándose en desarrollos teóricos y metodológicos basados en un enfoque interdisciplinario (Mexico in the modern, 2005).

La transformación del sistema internacional a inicios del siglo xxi contribuyó a una participación más activa de la región latinoamericana en los asuntos globales, lo que supuso para Rusia un renovado interés por comprender el lugar y el papel de la región latinoamericana y, en concreto, de México, en el sistema de las relaciones económicas, políticas y diplomáticas mundiales. 
La redistribución radical del orden establecido de interacción internacional, que condujo, entre otras cosas, a la entrada del sistema de relaciones interestatales en el hemisferio occidental en una nueva etapa de transformación, marcó una nueva senda caracterizada por la necesidad de nuevos estudios integrales que revelarían el contenido de las principales direcciones de las actividades de política exterior y económica exterior de México en el contexto del proceso histórico general. Esto ha llevado a la publicación, en los últimos años, de una serie de publicaciones importantes dedicadas a México y a los aspectos regionales y funcionales de su política exterior, entre la que cabe destacar Meksika: paradoksy modernizatsii, publicado en 2013 por el Instituto de América Latina de la Academia de Ciencias de Rusia (ILA RAS), así como Mexico in the System of Geopolitical Coordinates of the Beginning of the 21st Century (Kosevich, 2020), publicada por la Universidad Nacional de Investigación Escuela Superior de Economía.

Cabe señalar que Rusia también sigue atrayendo el interés científico de los principales expertos latinoamericanos. Estudios dedicados a la URSS y, posteriormente a la Federación Rusa, declarada su sucesor legal, han sido una parte integral de la ciencia académica de los países latinoamericanos desde la década de 1960. Los factores históricos han predeterminado en gran medida el desarrollo de estudios sistemáticos e integrales dedicados a las relaciones interregionales en general y, en particular, las relaciones entre Rusia y México. De especial trascendencia en este contexto son las publicaciones de la colección Archivo Histórico Diplomático Mexicano, dedicadas a la historia de las relaciones bilaterales en diferentes etapas (Ortega y Sizonenko, 1990).

La gran mayoría de los autores están de acuerdo en que, para Rusia, como un rival estratégico, militar e ideológico de Estados Unidos, la situación geopolítica de México no sólo era codiciada en tiempos de guerra, sino también en momentos de incertidumbre e inestabilidad institucional en el resto de América Latina (Cárdenas, 1993; Martínez y Garza, 2013). La principal explicación del interés de Moscú por acercarse a México, no únicamente durante la guerra fría, sino a lo largo de la historia contemporánea, reside en la excepcional situación del territorio mexicano, a través del cual podrían llevarse a cabo actividades de subversión en el resto del continente (Martínez, 2010).

Cabe señalar el aporte de investigadores mexicanos como Humberto Garza Elizondo y Susana Chacón, cuyas principales líneas de investigación incluyen la historia de las relaciones internacionales y la política exterior de México (Garza y Chacón, 2002). En una de sus obras, Humberto Garza planteó una serie de aspectos necesarios para comprender la naturaleza de las relaciones entre la URSS y México. Primero, "la asimetría" desarrollada entre la URSS, que ocupa el lugar de una de las dos superpotencias, aspirando a ser la primera, y México, que obtuvo el estatus de potencia media con una política exterior declarativa y marcadamente bilateral. En segundo término, la "baja prioridad", según la cual la URSS ocupa un lugar marginal en la escala de las relaciones internacionales de México, exactamente el mismo lugar secundario en la agenda internacional de la URSS que ocupa México. Por último, el simbolismo, que definía que las transacciones en cualquier nivel (político, económico o cultural) entre la URSS y México tenían un carácter marcado simbólico, siendo escasas e irregulares. No obstante, Garza (1988) señala que en el diálogo bilateral permanecen los vínculos y canales de comunicación, enfatizando que todavía existe una posibilidad real de que se desarrollen en el futuro. Este enfoque no ha perdido su relevancia en la etapa actual, lo que permite evaluar las relaciones actuales entre Rusia y México a través del prisma de estas tres características.

Varios artículos de autores latinoamericanos (Patiño, 2014; Picarella y Scocozza, 2018; Santos, 2010) están dedicados a la política de extensión de colaboración de Rusia con los países de América Latina, que comenzó a principios del siglo xxI, así como a la búsqueda de nuevas formas 
de influencia en esta región. A pesar de esto, la literatura científica más actual, ligada a los estudios sobre las relaciones Rusia-México modernas, abordan la temática desde una visión fragmentada. La investigación que se presenta pretende llenar el vacío existente, a través de un análisis exhaustivo de las nuevas tendencias en la cooperación económica externa entre los dos países a través de la rama de la historia económica.

Así pues, el objeto de investigación pasa por analizar la dinámica del desarrollo de las relaciones ruso-mexicanas en el contexto de los cambios en el entorno político y económico interno y global a finales del siglo xx y principios del xxI.

En ese marco, el objetivo del estudio es revelar las características de las relaciones bilaterales entre Rusia y México mediante la definición de las características principales del proceso de evolución de la cooperación económica en el periodo 2000-2019, considerada como un fenómeno multifactorial, e identificar las perspectivas de desarrollo. Para ello, las áreas humanitarias y culturales de cooperación brindan una imagen integral del diálogo bilateral.

En cuanto a la hipótesis de investigación, esta investigación es consciente del estado actual de las relaciones ruso-mexicanas, paradójico en muchos sentidos: por un lado, el alto nivel de diálogo político, coincidencia o similitud de posiciones en la mayoría de los temas de actualidad de la política mundial y amplia práctica de cooperación humanitaria; por otro, la escala de los lazos comerciales y económicos es extremadamente insignificante en comparación con los mayores Estados de América Latina. A pesar de que el potencial de cooperación respecto a varias posiciones se realiza parcialmente, Rusia continúa estando en la periferia de los intereses de política exterior de México. Mientras que el papel asignado a México en la "escala" de las prioridades de la política exterior rusa aún es muy bajo. Las relaciones bilaterales entre los dos países continúan caracterizándose por tres aspectos: asimetría, baja prioridad y simbolismo, lo que demuestra un alto grado de continuidad en relación con el siglo xx.

La base empírica de este artículo se basa en un compendio de fuentes abiertas y literatura científica sobre la política exterior, la política económica exterior de Rusia y México y de las relaciones ruso-mexicanas: documentos programáticos básicos de política exterior de Rusia y México, discursos de líderes clave de política exterior de los dos países, informes de medios y agencias de noticias, así como artículos académicos y libros de autores rusos y latinoamericanos, dedicados a las relaciones entre los dos países en la etapa actual y su desarrollo en el futuro previsible.

El análisis estructural forma la base del conjunto de herramientas de investigación metodológica. Se presupone la consideración del objeto (en este caso, las relaciones ruso-mexicanas) como un sistema, la identificación de sus elementos, las conexiones entre ellos y las propiedades básicas, principalmente la estructura. La necesidad de analizar las actividades de varias administraciones presidenciales predeterminó el uso del método histórico comparativo.

\section{El MARCo DE RELACIONES}

Con una mirada retrospectiva en el pasado reciente, debería reconocerse que la década de 1990 en su conjunto resultó de muy poco provecho para Rusia en las relaciones con Latinoamérica, principalmente a causa de dos factores. En primer lugar, la avalancha de información negativa sobre la realidad de la vida cotidiana en Rusia, que literalmente cayó sobre América Latina a través de los medios de comunicación occidentales, a principios de los años noventa, en apoyo a la consolidación de los estables estereotipos malignos y peyorativos de la percepción del país. En segundo lugar, las ininteligibles acciones de Rusia en el ámbito internacional, y en particular en la región 
latinoamericana, que favorecieron la formación de una imagen fundamentalmente desfavorable. Después de la disolución de la URSS, y ante los ojos de los Estados latinoamericanos, en el lugar de una superpotencia poderosa surgió un Estado incomprensible que anunció la transición a una asociación estratégica con Estados Unidos y comenzó un desmantelamiento caótico de sus posiciones en el tercer mundo.

La atención de casi toda América Latina, en primer lugar, se centró en Cuba, país con el que Rusia congeló las relaciones en gran escala (el intercambio comercial durante el periodo 19901993 disminuyó casi 30 veces). Cabe señalar que, durante este periodo histórico, una serie de acciones de política exterior de Rusia creó la imagen de un país impredecible que, además, buscaba el patrocinio político de Washington: los viajes escandalosos y políticamente inútiles de los parlamentarios rusos a Argentina, Chile y Ecuador en 1994, el inicio desastroso de la primera guerra chechena (1994-1996), la retirada mediocre de tropas rusas de Europa del Este, una reacción lánguida a la expansión de la Organización del Tratado del Atlántico Norte (OTAN), multiplicando con ello la imagen negativa de Rusia en el mundo, y en América Latina en particular.

Desde la perspectiva de la historia económica debe tenerse en cuenta que los primeros empresarios rusos en aparecer en los mercados latinoamericanos, absolutamente ajenos al entorno local, buscaron principalmente formas de obtener dinero rápido, lo que no les proporcionó una reputación adecuada. Las exrepúblicas soviéticas, a pesar de la formación de la Comunidad de Estados Independientes (CEI) en 1992, no sólo no trataron de coordinar sus pasos de política exterior en la región, sino que a menudo competían entre sí.

A principios de 1993 se publicó por primera vez la doctrina de política exterior rusa, en la que América Latina, por costumbre, entre las principales referencias geográficas a considerar, ocupaba el último lugar después de África. A comienzos de siglo, acciones casi extravagantes, con escasos referentes en la diplomacia moderna, tuvieron lugar en la práctica de la política exterior rusa en la dirección latinoamericana. Un caso ilustrativo de esa errática situación se puede encontrar en 1995, cuando la visita a México del ministro de Relaciones Exteriores, A. Kozyrev, se anunció en cinco ocasiones y, en el último momento, sin razones de peso, se canceló. Ello causó una reacción razonablemente negativa de las elites mexicanas.

El punto de inflexión llegó a principios del siglo xxi, con el final de la era de Boris Yeltsin (1991-1999). Los propios Estados latinoamericanos contribuyeron, en parte, por su comportamiento en el ámbito internacional. En 1999, el Grupo de Río era, de hecho, el único en el mundo que condenó el bombardeo de Yugoslavia, al indicar en su declaración qué artículos específicos de la Carta de las Naciones Unidas habían sido violados por los países de la OTAN. En febrero de 2003, México y Chile, siendo miembros no permanentes del Consejo de Seguridad de las Naciones Unidas, y a pesar de su dependencia económica de Estados Unidos, no sólo se opusieron a la segunda resolución angloamericana que autorizaba la intervención en Irak, sino que también reunieron a otros miembros no permanentes de países de Asia y África. Estas acciones hicieron que el Kremlin tomara una nueva postura ante las perspectivas de cooperación con los Estados de esta región. Al mismo tiempo, se percibió una especie de retorno de Rusia a América Latina, en gran medida asegurado por la "deriva izquierda" en la región, que condujo a la aparición de un grupo más o menos compacto de Estados que consideraba la expansión de los lazos con Rusia como una palanca importante para fortalecer sus posiciones, habida cuenta de las condiciones de las relaciones de conflicto con Estados Unidos. 
A principios de la tercera década del siglo xxi Rusia mantiene relaciones diplomáticas con los 33 países de América Latina y el Caribe, pero la dirección mexicana en la política exterior rusa aún no ha adquirido un carácter estratégico e independiente.

Los 131 años transcurridos desde el establecimiento de las relaciones oficiales entre Rusia y México, en diciembre de 1890, se han caracterizado por presentar acusados altibajos en el diálogo bilateral sobre una amplia variedad de temas. Las relaciones diplomáticas entre México y Rusia pasaron por varias etapas: fueron interrumpidas en 1914 y restauradas en 1924, ya con la Unión de Repúblicas Socialistas Soviéticas (URSS); cortadas en 1930 y restauradas nuevamente en 1943. En cada una de estas etapas se apreciaba una recurrente cooperación multifacética, notando las perspectivas prometedoras, sin tener en cuenta la situación internacional emergente y la coyuntura política. A pesar de las numerosas diferencias culturales entre los dos países, así como de las vastas distancias que los separan, se pueden notar varios episodios bastante similares que dejaron impronta en la historia de estos países: el periodo de privatización masiva de empresas estatales (en la literatura rusa se encuentra a menudo el término salinastroyka con referencia a perestroika ${ }^{1}$ ), la transición hacia el libre comercio, y la búsqueda interrumpible de las formas de salir de las crisis económicas subsiguientes (Borovkov, 1999). Como resultado, a finales del siglo xx, tras cambiar casi simultáneamente a nuevos modelos de desarrollo económico y político, en 2019 Rusia y México se han convertido en países de importancia comparable en sus regiones (Davydov, 2016).

México ha demostrado la transición a un nuevo modelo de desarrollo, descrito por los principales especialistas rusos en América Latina como un "giro en la historia", lo que, en definitiva, lo ha llevado a convertirse en uno de los países más influyentes de América Latina. Como resultado del intenso diálogo diplomático y político de la segunda década del siglo xxi, México y Rusia han ocupado de forma predominante posiciones similares en programas de cooperación multilateral bajo la Organización de las Naciones Unidas, Grupo de los 20, Foro de Cooperación Económica Asia-Pacífico (APEC) ${ }^{2}$ y la Organización Mundial de Comercio (Davydov y Kalashnikov, 2006). Los intereses de estos países también coincidieron en tales materias, como la lucha contra el crimen organizado, el combate contra el terrorismo internacional y la protección del medioambiente (Martynov, 2014).

México tradicionalmente ha valorado positivamente el nivel de relaciones bilaterales que tenía con la Unión Soviética, que para el país azteca se convirtió en una especie de la confirmación de su autosuficiencia en el diálogo de la política exterior contemporáneo, así como una cierta demostración de su autonomía de Estados Unidos. Tales hechos quedaron demostrados en las visitas regulares de los presidentes mexicanos a Moscú (Borovkov et al., 2005). El alto grado de interés de México hacia la URSS lo distinguía favorablemente entre todos los países latinoamericanos, compitiendo solamente con Cuba.

Apenas unas semanas después de la visita de Estado del presidente mexicano Carlos Salinas (1988-1994) a Moscú, en julio de 1991, tuvo lugar el Putch de agosto, que puso en marcha procesos políticos, cuyo principal resultado fue que la Unión Soviética dio paso a la Federación Rusa. Casi inmediatamente después de los trágicos acontecimientos ocurridos en la capital rusa, el mandatario mexicano envió su mensaje al primer presidente de la Federación de Rusa, Boris Yeltsin (1991-

${ }^{1}$ La Perestroika (en ruso reestructuración) es conocida como la reforma económica destinada a desarrollar una nueva estructura de la economía interna de la Unión Soviética.

${ }^{2}$ Siglas en inglés de Asia-Pacific Economic Cooperation. El APEc es un foro multilateral creado en 1989 con el fin de consolidar el crecimiento y la prosperidad de los países alrededor del Pacífico, que trata temas relacionados con el intercambio comercial, coordinación económica y cooperación entre sus integrantes. 
1999), en el cual se refiere a la reunión celebrada sólo unos días antes, y reitera nuevamente "la importancia de fortalecimiento de las relaciones entre Rusia y México, en el contexto de intensos cambios estructurales que tienen lugar en ambos estados" (Sizonenko, 2004, p. 53).

A pesar de esto, después de la caída de la URSS las relaciones bilaterales han cambiado notablemente. Al perder su único punto de apoyo en el mundo con la naciente hegemonía estadunidense, Los Pinos ${ }^{3}$ miraban hacia la nueva Rusia con reproche, pero ya sin interés (Simonova, 2013). Como resultado de todo ello, desde 1995 las conexiones diplomáticas prácticamente han dejado de desarrollarse.

El tan esperado acercamiento diplomático comenzó a formarse a principios del siglo xxi. Las importantes visitas de Estado realizadas en 2004 y 2005, cuando primero el presidente ruso Vladimir Putin (2000-2008, 2012-presente) viajó a la ciudad de México, y un año después, el presidente mexicano Vicente Fox (2000-2006) correspondió con su visita a la capital rusa. La llamada Iniciativa Rusia-México: hacia una Nueva Era de Cooperación, una declaración conjunta firmada en México, constató la importancia y el potencial de los lazos comerciales y económicos existentes entre ambos Estados. Como parte de la primera visita del presidente ruso a tierras mexicanas, en la historia de la nueva Rusia se delinearon nuevas direcciones para el desarrollo futuro de las relaciones entre los dos países. En ese sentido, la visita de Putin a México en 2004 permitió ampliar significativamente el rango de las relaciones diplomáticas, pero no logró amplificar la cooperación en el campo comercial. Esto se debió, principalmente, al hecho de que los proyectos anunciados a gran escala no han sido realizados. En concreto, la parte rusa propuso un proyecto para el suministro de gas licuado desde el Lejano Oriente hasta la costa del Pacífico de México, pero no dio ningún paso práctico en esta dirección, lo que ha desacreditado este proyecto. Este desencuentro sucedió en connivencia con un marco legal desactualizado, basado en documentos que se firmaron en la década de 1970 a 1980. La renovación de esta base se vio obstaculizada en gran medida por las negociaciones incompletas (hasta el 2013) sobre la adhesión de Rusia a la Organización Mundial del Comercio (OMC). ${ }^{4}$

La visita del presidente Fox a Moscú en 2005 culminó con la firma de una serie de acuerdos en las áreas de inversión, comercio, energía y tecnología. A pesar de que la parte mexicana confirmó su disposición a apoyar la adhesión de Rusia a la omc, en aquel momento los procedimientos antidumping se aplicaron repetidamente a los exportadores rusos, confirmando que Rusia se percibía en la región como un país con una "economía no de mercado".

A pesar de esto, después de 2005, en las relaciones ruso-mexicanas nuevamente surgió una pausa. A principios del siglo xxi Rusia estaba expandiendo activamente su presencia en varios países latinoamericanos, tanto en forma de relaciones bilaterales, como de cooperación multilateral. Sin embargo, fue México, entre todos los Estados latinoamericanos, el que se encontró al margen del mencionado acercamiento político activo. En comparación con otros países latinoamericanos con los que Rusia intercambiaba regularmente visitas de alto nivel político, en las que participaban los jefes de Estado denotando las perspectivas reales para el desarrollo de las colaboraciones, en

${ }^{3}$ El Complejo Cultural Los Pinos, anteriormente Residencia Oficial de Los Pinos, y referido coloquialmente como Los Pinos, fue la residencia del presidente de los Estados Unidos Mexicanos desde 1934 hasta el 30 de noviembre de 2018. Desde el 1 de diciembre de 2018 es un espacio cultural abierto al público dentro del Bosque de Chapultepec.

${ }^{4}$ Es la única organización internacional que se ocupa de las normas que rigen el comercio entre los países. Los pilares sobre los que descansa son los Acuerdos de la omc, que han sido negociados y firmados por la gran mayoría de los países que participan en el comercio mundial y ratificados por sus respectivos parlamentos. Su objetivo principal es ayudar a los productores de bienes y servicios, los exportadores y los importadores a llevar adelante sus actividades. 
el caso de México esto se limitó con las visitas de titulares de la Secretaría de Relaciones Exteriores y otros departamentos. Los contactos bilaterales entre los jefes de Estado de Rusia y México tuvieron lugar "en los campos" de las cumbres internacionales. Ni por parte de México a Rusia, ni tampoco por parte de Rusia a México, durante la última década y media no se ha realizado ninguna visita oficial completa, lo cual puede ser considerado como una omisión muy seria para las relaciones bilaterales. Esto llevó al hecho de que, a principios de la tercera década del siglo xxI, México, en comparación con otros países latinoamericanos, había extraviado manifiestamente su presencia en la política exterior de Rusia. A su vez, Rusia ha perdido su lugar en la política exterior de México.

Sin tomar en consideración la llegada a San Petersburgo en 2013, en el marco de la cumbre del G-20, de Enrique Peña Nieto (2012-2018), la última visita oficial del líder mexicano tuvo lugar en 2005, cuando el presidente Vicente Fox visitó Moscú. Y sólo catorce años después el nuevo presidente de México, Andrés Manuel López Obrador (2018-presente), aceptó la invitación para hacer una visita a Rusia, dando así razones para esperar el desarrollo acontecido de la cooperación tan esperado.

Pese a que ya se había señalado, a principios del siglo xxi los contactos a nivel de jefes de cancillerías se estaban desarrollando mucho más activamente, en octubre de 2008 la entonces secretaria de Relaciones Exteriores de México, Patricia Espinosa, realizó una visita oficial a Moscú, en la cual se reunió con su homólogo Sergey Lavrov y la secretaria de Desarrollo Económico de Rusia, Elvira Nabiullina. En el marco de su visita, Espinosa confirmó el apoyo necesario de México para la adhesión de Rusia a la omc y haber obtenido el compromiso del voto de Moscú a favor de la candidatura de México para el puesto de miembro no permanente del Consejo de Seguridad de las Naciones Unidas para el periodo 2009-2010.

En febrero de 2010, Sergey Lavrov volvió a visitar México para discutir posibles perspectivas de la intensificación de la cooperación bilateral en áreas como la económica, cultural y científica. El resultado más importante de las negociaciones realizadas fue la firma, por parte de las Secretarías de Relaciones Exteriores, del Programa de Cooperación Científica y Técnica entre la Federación de Rusia y México para el periodo 2011-2013, en el marco del cual se planeaba implementar 18 proyectos conjuntos, afectando los campos de nanotecnología, aeronáutica y biotecnología (Davydov, 2003). Finalmente, en 2011 el gobierno mexicano reconoció que Rusia ya estaba inmerso en el sistema de economía de mercado. Todo esto ha llevado al hecho de que, desde principios del siglo XxI, el intercambio comercial entre los dos países ha aumentado significativamente, llegando a casi 1900 millones de dólares en 2013. Además, se han logrado ciertos avances de la participación empresarial rusa en el campo de la cooperación energética. Por poner un ejemplo de este renovado impulso de colaboración, la empresa rusa de construcción de maquinaria energética Power Machines PJS participó en la instalación de la central hidroeléctrica mexicana La Yesca.

Tras los resultados de la primera década del siglo xxi se puede notar que, en su mayor parte, los enfoques de ambos países a los principales problemas internacionales se caracterizaron por un cierto acercamiento de puntos de vista, aunque se habían detectado ciertas excepciones.

Esto se ha manifestado en la evaluación de la adhesión de Crimea a la Federación de Rusia, cuando México -junto con España y otros países latinoamericanos como Chile, Colombia, Costa Rica, República Dominicana, Guatemala, Haití, Honduras, Panamá y Perú- votó a favor de la resolución de la Asamblea General de las Naciones Unidas sobre la integridad territorial de Ucrania, donde fue reconocido el referéndum de Crimea como inválido. Pero al mismo tiempo, México 
se abstuvo de criticar públicamente las acciones de Moscú, a diferencia de Madrid, quien declaraba públicamente que Rusia era una de las amenazas más graves para la seguridad internacional. Aparte de eso, los medios locales mexicanos cubrieron los detalles de este episodio político con poca intensidad, lo que, en general, no ha cambiado la imagen de Rusia en calificación de la población mexicana. Cabe señalar que el Ministerio de Asuntos Exteriores de Rusia reconocía que la posición negativa de México sobre el tema de Crimea se debió en gran parte al hecho de que, en el siglo xIx, Estados Unidos “cortó” y anexó más de la mitad del territorio mexicano.

Cabe destacar la reacción de la Secretaría de Relaciones Exteriores de México al caso de Skripals, ${ }^{5}$ donde se decidió anunciar solamente las frases neutrales de su "preocupación" respecto al uso de armas químicas como un ejemplo de violación de la Convención sobre Armas Químicas y las normas del derecho internacional, enfatizando la necesidad de una investigación independiente. Al mismo tiempo, esta posición estaba destacándose por su carácter bastante pasivo entre otros comentarios por parte de la mayoría de los países latinoamericanos, los cuales eran claramente acusatorios.

Una divergencia de opinión mucho más notoria estuvo presente en las discusiones sobre la reforma del Consejo de Seguridad de las Naciones Unidas (Kuteinikov, 2012). México, junto con Francia, tomó la iniciativa de limitar el uso del veto por parte de los miembros permanentes del Consejo de Seguridad, la cual, finalmente, ha sido apoyada por más de 110 países. A su vez, Rusia continuó expresando su desacuerdo con cualquier propuesta destinada a limitar las prerrogativas de los miembros permanentes del Consejo de Seguridad, incluso el mecanismo de veto (Kosevich, 2017).

En 1994 México se convirtió en el primer miembro latinoamericano de la Organización para la Cooperación y el Desarrollo Económicos (OCDE). Además de eso, el cargo de secretario General de la OCDE desde 2006 ha sido ocupado por el extitular de la Secretaría de Relaciones Exteriores, José Ángel Gurria, quien había visitado previamente Moscú de manera oficial. Al mismo tiempo, a pesar de la solicitud de Rusia de unirse a la OCDE, expresada aún en 1995, en 2014 el Consejo de esta tomó la decisión final de suspender el proceso de adhesión de Rusia a la Organización, otorgándole sólo el estatus de observador.

Es importante señalar la ambiciosa participación de los mexicanos respecto a la discusión del tema del desarme nuclear y la seguridad internacional. México, al ser el principal promotor del Tratado de Tlatelolco firmado en 1967, que convirtió a América Latina en una zona libre de armas nucleares, sigue sosteniendo una opinión de que todavía no hay un acuerdo absoluto sobre cuestiones de desarme, reconociendo abiertamente el diálogo corriente sobre este tema como extremadamente vago (Zaemsky, 2008). Los resultados de la IX Conferencia de Revisión de las Partes del Tratado sobre la No Proliferación de Armas Nucleares (TNP), celebrada en mayo de 2015, una vez más confirman la inhibición del proceso de creación de Zonas Libres de Armas Nucleares en el mundo (Primakov, 2014). Lo que, entre otras cosas, implica nuevas consultas activas entre Rusia y México sobre no proliferación nuclear y desarme, dado el hecho de que Medio Oriente continúa siendo una región de inestabilidad nuclear (Patrick, 2014).

${ }^{5}$ El caso del envenenamiento del ex agente ruso Sergei Skripal y su hija Yulia, que tuvo lugar el 4 de marzo de 2018 en el condado de Salisbury, Wiltshire, Inglaterra, Reino Unido. El gobierno del Reino Unido acusó a Rusia de estar involucrada en el intento de asesinato de los Skripals y en violación de la Convención de Armas Químicas. Moscú categóricamente rechazó estas acusaciones y declaró que el envenenamiento fue una provocación que podría haber sido organizada por los servicios especiales de Gran Bretaña o Estados Unidos. Dicho incidente hizo estallar un conflicto diplomático, que ha provocado una reacción masiva de expulsiones de diplomáticos rusos de países occidentales. 
La expansión del diálogo bilateral sobre cuestiones de política mundial fue facilitada por la actividad de la Comisión Bilateral Ruso-Mexicana de Consultas sobre Cuestiones Multilaterales, ${ }^{6}$ que ha celebrado varias reuniones. Entre los temas principales del trabajo de la Comisión, junto con los problemas de desarme, se encuentran los mecanismos para la protección de los derechos humanos a nivel mundial, en particular en el marco de la participación de ambos Estados en el trabajo del Consejo de Derechos Humanos de las Naciones Unidas (Martynov, 2017).

Finalmente, es importante destacar que, para la Unión Soviética, el país azteca desempeñaba el papel de "aliado político-concepcional" en la región: a pesar de las diferencias en las ideologías dominantes, los regímenes políticos y los sistemas económicos, ambos países han podido encontrar puntos en común al discutir temas pendientes de la política mundial, lo que distinguía ventajosamente a México de entre otros países latinoamericanos. Para la Federación de Rusia, que utilizaba la memoria histórica de los Estados latinoamericanos para aumentar la influencia en el hemisferio occidental, México se había visto como un "socio económico potencial". El énfasis principal en el diálogo con México se coloca precisamente en la promoción del comercio, la cooperación económica, cultural y humanitaria, así como la consolidación de las empresas rusas en sectores industriales dinámicos. La expansión de la colaboración política continúa ocupando un lugar secundario En este contexto, México desempeña actualmente "el papel compensable" en el posicionamiento de Rusia en América Latina.

\section{Colaboración ECONÓMica}

México es uno de los socios comerciales tradicionales de Rusia en América Latina. Sin embargo, hasta el 2020, el volumen de comercio mutuo no era estable, y los periodos de crecimiento a menudo se sucedían por lapsos de descenso y estancamiento. Los signos de un punto de inflexión en ese aspecto concreto se han delineado en los últimos años, pues los parámetros cuantitativos y, en cierta medida, cualitativos de los lazos comerciales y económicos han aumentado. Al mismo tiempo, la cuestión de la sostenibilidad de esta tendencia sigue abierta.

Desde finales del siglo pasado, en la agenda de las relaciones bilaterales está pendiente la cuestión de abrir una nueva línea de transporte a través del océano Pacífico. La membresía de Rusia y México en el APEc brinda oportunidades favorables para esto. En el foro empresarial del Pacífico, celebrado en Vladivostok en 1990, la delegación mexicana que participó en él expresó su deseo de invertir en la infraestructura del Territorio de Primorie, pero la desaparición de la URSS en 1991 eliminó este tema de la agenda.

Una participación notable en el desarrollo de los lazos en el siglo xxı lo desempeñó la cooperación en el campo de la electricidad. Entre los años de 1992 y 1995 Rusia participó en la construcción de las centrales hidroeléctricas mexicanas. De más reciente factura, se puede mencionar la colaboración en el suministro del equipo hidroeléctrico de la compañía rusa Power Machines PJS, la cual ha sido certificada como un proveedor confiable por la Comisión Federal de Electricidad de México.

A finales de la segunda década del siglo xxi el nivel de cooperación económica entre ambas naciones todavía sigue sin alcanzar el nivel potencial entre ambos países. Una etapa que puede ser evaluada como revolucionaria, al menos en lo relativo a esta área, comenzó con el gobierno de

${ }^{6}$ Comisión Bilateral Ruso-Mexicana de Consultas sobre Cuestiones Multilaterales fue creada en 1996 con el objetivo de incrementar los flujos comerciales y de inversión. 
Enrique Peña Nieto (2012-2018). El presidente Peña estableció el objetivo de reforzar significativamente los lazos económicos con Rusia. Bajo su administración, el centro de toda la política exterior fue puesto "el principio de equidistancia", lo que representó una reorientación de México al dar la espalda a Estados Unidos y enfocarse en otras regiones, Rusia incluida. Tradicionalmente este principio es el concepto fundamental de política exterior del Partido Revolucionario Institucional. Pero bajo las administraciones de Vicente Fox y Felipe Calderón, ambos miembros del Partido Acción Nacional (PAN), este principio perdió su importancia, puesto que existió una preponderancia abrumadora a favor de las relaciones con Estados Unidos.

En mayo de 2015, en Moscú se abrió una oficina de ProMéxico, cuyo objetivo principal era la búsqueda de nuevas oportunidades para aumentar el comercio bilateral, así como proyectos potenciales para inversiones mexicanas en la Federación de Rusia. En enero de 2018 comenzó a funcionar en Moscú la Oficina de la Asociación Nacional de Importadores y Exportadores de la República Mexicana (ANIERM), a través de la cual los empresarios mexicanos planeaban establecer relaciones comerciales con representantes de empresas rusas, incluso con las que se ubican en las regiones rusas ajenas a la parte central del país.

A pesar de que México y Rusia presentan muchas oportunidades para el aumento del comercio bilateral, el intercambio comercial actual entre los dos países continúa manteniendo niveles extremadamente bajos. No obstante, los cambios positivos generados a principios del siglo xxi, caracterizados por el desarrollo progresivo de las relaciones económicas ruso-mexicanas, siguen sin resolver una amplia gama de problemas: $a$ ) el modesto volumen de comercio mutuo y desequilibrios comerciales; $b$ ) el lento proceso de reestructuración de la estructura de los productos básicos de las exportaciones rusas (para 2020 los productos básicos y los productos con una baja proporción de valor agregado dominan). Y esto contrasta con el hecho de que hace dos décadas México era un importante consumidor de maquinaria y equipos rusos, y $c$ ) el bajo nivel de interacción de inversión. Los problemas de las restricciones aduaneras también siguen sin resolverse. En particular, la aplicación de medidas antidumping en relación con algunos productos rusos (por ejemplo chapa de acero). La formación del marco regulatorio y legal es incompleta. El sistema de acuerdos mutuos y seguros de las operaciones de exportación e importación sigue siendo vulnerable. La cuestión sobre la mejora del clima de inversión también se está resolviendo lentamente. Según los empresarios mexicanos, el clima de inversión en la Federación de Rusia es menos favorable que en la mayoría de los países del mundo. Otra razón importante de esta situación es la falta de conciencia. Tradicionalmente, los empresarios rusos perciben a México como "un país de tequila, sombrero y las playas de Cancún", ignorando el hecho de que este país ya tiene una cartera de inversiones muy considerable. En lo que se refiere a Rusia, para los mexicanos la información sobre su cultura y sus tradiciones en la mayoría de los casos se obtiene de las películas de Hollywood. La imagen de una enorme, agresiva e incontrolada potencia mundial que suele tener dificultades en el establecimiento de nuevos lazos políticos y económicos, con mucho éxito se difunde gracias a los medios estadunidenses, de gran impacto en la sociedad mexicana. Todo esto confirma que las relaciones bilaterales comerciales continúan caracterizándose por la baja prioridad y el simbolismo, y no por un avance real.

En 2012, antes de que el presidente Peña llegara a Los Pinos, el volumen de inversiones directas rusas en México era "prácticamente cero". Pero desde el comienzo de su gobierno las inversiones de Rusia a México han alcanzado los 14400000 dólares anuales, lo que aún sigue siendo insignificante en comparación con la cantidad de la inversión proveniente de otros países 
en el mismo periodo. ${ }^{7}$ Sin embargo, incluso estas modestas cifras han marcado un cierto grado de aproximación e interés nunca antes vistos entre el Kremlin y Los Pinos: fueron significativamente más altos que las cifras finales de inversión que aparecían durante la administración del presidente Calderón, cuando la inversión rusa en México ascendió a sólo 2800000 dólares; así como las cifras finales de 800000 dólares en la presidencia de Fox, a pesar del hecho de que, justamente durante su presidencia, la anteriormente mencionada iniciativa ruso-mexicana había sido firmada.

En términos generales, durante el sexenio del presidente Peña Nieto la inversión rusa en México creció más de $400 \%$ en los sectores energético, agropecuario, de construcción y químico. Cabe señalar que, entre 1999 y 2018, ascendió a 39700000 dólares estadunidenses el volumen total de la inversión extranjera directa de Rusia en México, el aumento fue significativo principalmente en los sectores de la minería (55.9\%); transportes, correos y almacenamiento $(25.6 \%)$ y en los servicios de alojamiento temporal (14.9\%) (Federal State Statistic Service, 2020). En particular, en 2017 la empresa automotriz rusa LLK Internacional realizó un proyecto de inversión a gran escala en la industria automotriz en México. Además, empresarios mexicanos y rusos anunciaron una inversión por más de 15000000 de dólares en proyectos estratégicos de energía en México. En este contexto, el experto ruso N. Shkolyar confirma la existencia de oportunidades reales para profundizar la cooperación para las empresas rusas en los sectores de aviación, transporte automotor, minería y agricultura (Shkolyar, 2019).

Además, durante 2018 el crecimiento del intercambio mutuo de mercancías fue bastante remarcado (véase cuadro 1). Según los datos proporcionados por la Secretaría de Economía de México, desde 1997 el superávit comercial en el comercio bilateral se ha fijado firmemente a favor de Rusia. Durante el periodo 1996-2017 el volumen del comercio entre los dos países ha crecido considerablemente, pues comparando los datos de 1996, cuando el comercio bilateral fue de sólo 211000000 de dólares, con un déficit comercial de México de 92400000 dólares, en 2018 el comercio bilateral total alcanzó 2300 millones de dólares, con una balanza comercial desfavorable para México de 1600 millones de dólares. Durante el periodo indicado, el intercambio mercantil entre los dos países estaba aumentando gradualmente, desarrollándose con beneficios comerciales para Rusia.

Es de destacar que el volumen de comercio bilateral comenzó a aumentar después de la crisis financiera mundial de 2008-2009. Esto se debe al hecho de que México, estrechamente vinculado a la economía estadunidense como como ningún otro Estado, sintió el negativo impacto de esta crisis y estas condiciones. Así, el liderazgo mexicano se embarcó en una búsqueda activa de nuevos socios.

Importantes sectores de la industria rusa, como la maderera, la metalúrgica y la energética han empezado a ser de particular interés para México. También de Rusia a México se importan materias primas de minerales y granos, fertilizantes químicos y productos industriales. Las principales posiciones de las exportaciones mexicanas a Rusia son automóviles y piezas de recambio, así como bebidas alcohólicas, principalmente tequila y cerveza.

En los últimos años, la principal prioridad de México ha sido el aumento de las exportaciones de alimentos, aprovechando las nuevas oportunidades que se abrieron tras el embargo alimenticio impuesto por Moscú a productos procedentes de la Unión Europea y Estados Unidos, como res-

${ }^{7}$ Desde comienzos del siglo xxi, el promedio de las inversiones en México provientes de Estados Unidos es de más de 10000 millones de dólares anuales. 


\section{CUADRO 1. BALANZA COMERGIAL DE MÉXICO CON RUSIA (MILLONES DE DÓLARES)}

\begin{tabular}{|c|c|c|c|c|}
\hline Año & $\begin{array}{l}\text { Las exportaciones } \\
\text { de México a Rusia }\end{array}$ & $\begin{array}{l}\text { Importaciones de } \\
\text { Rusia a México }\end{array}$ & $\begin{array}{c}\text { Comercio bilateral } \\
\text { total }\end{array}$ & $\begin{array}{c}\text { La balanza comerciat } \\
\text { de México en el } \\
\text { comercio bilateral }\end{array}$ \\
\hline 1996 & 151 & 59 & 211 & 92 \\
\hline 1997 & 13 & 180 & 194 & -166 \\
\hline 1998 & 5 & 245 & 251 & -239 \\
\hline 1999 & 2 & 166 & 168 & -163 \\
\hline 2000 & 6 & 333 & 339 & -327 \\
\hline 2001 & 18 & 243 & 261 & -225 \\
\hline 2002 & 18 & 236 & 254 & -217 \\
\hline 2003 & 47 & 248 & 295 & -200 \\
\hline 2004 & 25 & 437 & 463 & -411 \\
\hline 2005 & 28 & 562 & 591 & -534 \\
\hline 2006 & 44 & 535 & 579 & -490 \\
\hline 2007 & 159 & 448 & 608 & -289 \\
\hline 2008 & 116 & 821 & 937 & -705 \\
\hline 2009 & 39 & 468 & 508 & -429 \\
\hline 2010 & 237 & 854 & 1092 & -617 \\
\hline 2011 & 471 & 1149 & 1620 & -677 \\
\hline 2012 & 490 & 1208 & 1699 & -717 \\
\hline 2013 & 472 & 1212 & 1684 & -739 \\
\hline 2014 & 274 & 1510 & 1784 & -1236 \\
\hline 2015 & 200 & 1543 & 1743 & -1343 \\
\hline 2016 & 178 & 1301 & 1480 & -1123 \\
\hline 2017 & 255 & 1461 & 1717 & -1206 \\
\hline 2018 & 327 & 1984 & 2312 & -1657 \\
\hline 2019 & 1107 & 1478 & 2585 & -371 \\
\hline
\end{tabular}

Fuentes: Balanza Comercial de Mercancías de México, años 1993-2018; Instituto Nacional de Estadística y Geografía. Información de Interés Nacional, año 2019; Statistics of Foreign trade of Russia, Federal Customs Service of Russian Federation.

puesta a las sanciones impuestas a Rusia por Occidente por el conflicto en Ucrania. Productos como mermeladas, chile, cacao, café, miel, salsas picantes y dulces se convirtieron en los principales productos alimenticios más vendidos a Rusia por las empresas mexicanas. Aunque los productos alimenticios representaron menos de $10 \%$ de exportaciones mexicanas totales a Rusia, México comenzó a mostrar un especial interés en promover sus frutas y verduras en el mercado ruso, especialmente el aguacate, cítricos y bayas, así como carne y productos lácteos. El restante $90 \%$ de las exportaciones de México a Rusia siguen manteniéndose de partes automotrices y piezas de recambio de aviones. 
A fines de 2018, México continuó negociando activamente con las autoridades rusas, esperando obtener la autorización de los certificados fitosanitarios necesarios para la exportación de carne y productos lácteos mexicanos a Rusia. Teniendo en cuenta que muchas empresas mexicanas ya estaban suministrando sus productos a Estados Unidos y Canadá durante las últimas décadas, lo que puede confirmar su cumplimiento con los estándares de calidad de estos países. Esa experiencia, sin duda, puede favorecer a las empresas mexicanas para ingresar con éxito al mercado ruso en un tiempo cercano. En general, el volumen del comercio bilateral entre México y Rusia en comparación con los datos de fines del siglo xx ha aumentado doce veces, con un crecimiento anual de 17 por ciento.

Si en 2012 en México estaban registradas 52 empresas con capital ruso, a finales de 2017 ya había 79 empresas rusas, cuyas actividades se desarrollan en áreas como aeronáutica, energía, hotelería, telecomunicaciones y tecnología de la información. En México, en los últimos años se hace cada vez más evidente la presencia de Rosoboronexport, una empresa rusa estatal responsable de suministros de helicópteros y vehículos blindados de fabricación rusa para las Fuerzas Armadas Mexicanas. Entre los planes de este mediador estatal en el campo de la cooperación técnico-militar está acordar los suministros de equipos militares para las fuerzas terrestres y equipos especiales para los servicios policiales a México en un futuro próximo. En abril de 2017, Rosoboronexport presentó por primera vez las muestras de sus productos en el salón aeroespacial mexicano Famex, lo que confirma la tendencia positiva en esta área. Sin embargo, todavía es muy poco probable que México alcance el mismo nivel de cooperación técnico-militar con Rusia en el que ya se encuentran Venezuela, Nicaragua y Cuba (Suárez, 2015).

Además, en 2015 México compró 30 aviones rusos Sukhoi Superjet 100 (S.S.100) más, tomando en cuenta que las primeras adquisiciones de este modelo por parte de los mexicanos comenzaron en 2012. La línea aérea mexicana Interjet se convirtió así en el segundo mayor comprador de aviones S.S.100 a nivel internacional, después de Aeroflot (la compañía aérea más grande de Rusia), la cual ha expresado una voluntad de edificación en el futuro próximo de una empresa conjunta con la compañía rusa Sukhoi Civil Aircraft, que va a especializarse en producción de piezas de repuesto para este modelo de avión. A finales de 2017, en medios masivos de comunicación, apareció la información de que el gobierno mexicano estaba interesado en el establecimiento en México de una gran fábrica de la empresa Helicópteros Rusos, cuyos bienes producidos estarían destinados al mercado latinoamericano.

En 2017 el fabricante de automóviles ruso Ulyanovsk Automobile Plant suministró a México 87 vehículos todoterreno de modelos Hunter y Patriot. Además, en un futuro cercano se planea efectuar los suministros de otros 134 automóviles más. Moscú ya había expresado la idea de crear en México un hub estratégico para la industria automotriz, lo cual permitirá a Rusia suministrar automóviles a aquellos países latinoamericanos con los que México ya ha establecido relaciones comerciales estables.

En el verano de 2016, cuando Donald Trump se convirtió oficialmente en un candidato presidencial del Partido Republicano con una campaña electoral caracterizada por las declaraciones antimexicanas, Rusia invirtió 1700000 dólares en la fabricación de tuberías para transportación y almacenaje del gas natural en doce estados mexicanos. En 2017, la inversión rusa en tuberías creció casi cinco veces y ascendió a 9800000 dólares.

México es uno de los mayores productores mundiales de petróleo, importadores de gas y productos derivados del este. En 2013, la administración del presidente Peña Nieto llevó a cabo una reforma del sector energético, que desmonopolizó este sector, lo que abrió el acceso al capital pri- 
vado y extranjero a su participación en todos los procesos, desde la extracción de petróleo hasta su distribución (Rogozhin, 2016). A finales de la segunda década del siglo Xxi, al mando de las áreas prioritarias y prometedoras de cooperación bilateral Rusia-México, cuyas reservas recuperables se estiman en 115000 millones de barriles de petróleo crudo equivalente, estaba la colaboración de la petrolera rusa Lukoil y la petrolera estatal mexicana Pemex en materia de exploración, desarrollo y producción de crudo. En 2018 la compañía rusa Lukoil, junto a la italiana Eni, ganaron la licitación para desarrollar la sección de hidrocarburos 28 en la provincia de Cuencas del Surarest, en las aguas costeras del Golfo de México. Ambos países mostraron mucho mayor interés en firmar un tratado comercial entre México y la Unión Económica Euroasiática, cuyos países miembros son Rusia, Bielorrusia, Kazajstán, Armenia y Kirguistán.

A finales de 2017 -en el curso de su participación en el foro de negocios Diálogo ComercioIndustrial: Rusia México- Denis V. Manturov, ministro de Industria y Comercio de la Federación de Rusia, enfatizó el interés de Moscú por celebrar un acuerdo bilateral de comercio e industria, que tiene como propósito brindar beneficios económicos especiales a los inversores en tecnología.

Vale la pena mencionar especialmente el interés de los turistas rusos de viajar a México. En 2013 se registró un récord cuantitativo con la mayor cantidad de turistas rusos de 120000 personas que visitaron México, en comparación con el año 2010, en el cual se contabilizaron sólo 11000 turistas. Pero la situación económica bastante difícil de México, así como la renuencia de proporcionar subsidios a los operadores turísticos y aerolíneas rusos que están trabajando en esta ruta, han causado que el número de turistas rusos se redujera.

En 2017 México experimentó el verdadero auge del turismo, después de haber acogido casi 40000000 de turistas y haber convertido esta industria en la tercera fuente de los ingresos del país, después de la industria automotriz y las remesas de los trabajadores mexicanos a sus familias. Pero de esta gran cantidad de turistas habían llegado tan sólo 30000 rusos, el mismo número que dos años anteriores. Con todo eso, la embajada de México en la Federación de Rusia continúa promoviendo activamente los viajes a las playas mexicanas. En este contexto, en noviembre de 2014, y con el objetivo de atraer a los turistas rusos a los destinos turísticos, así como intensificar las negociaciones con las aerolíneas sobre la organización de nuevos vuelos chárter, se abrió la primera oficina de representación del Consejo Mexicano de Promoción del Turismo. Cabe señalar que la guerra del narco iniciada en México en 2006, la cual, según los últimos datos, cobró la vida de más de 250000 personas, incluido un número considerable de extranjeros, afectó la imagen de los famosos centros turísticos mexicanos.

En lo que se refiere a Rusia, este país sigue siendo un lugar extremadamente impopular para los turistas de México: en 2017 el número total de turistas era de 4800 000, de los cuales 16000 eran mexicanos, no obstante, el crecimiento fue modesto respecto a los 8000 turistas de 2010 . Los datos de 2018, incluso teniendo en cuenta la pasada Copa Mundial de la FIFA, en términos de la cantidad de turistas que han visitado Rusia, México retuvo sólo el lugar 50, puesto que ha ocupado invariablemente durante varios años consecutivos. Las principales razones identificadas para esta situación son la distancia y la inexistencia de vuelos directos entre los dos países, lo que provoca costos de desplazamiento muy altos para los turistas mexicanos. En 2012 el liderazgo mexicano estableció un objetivo ambicioso de aumentar el número de turistas de Rusia a 500000 personas para 2018, sin embargo, nunca se realizó. 


\section{COOPERACIÓN HUMANITARIA Y GULTURAL}

En cuanto al vector humanitario de las relaciones, la transición hacia un nuevo nivel de cooperación bilateral entre México y Rusia en el campo de la ciencia, la tecnología y la educación se ha desarrollado después de la firma del acuerdo intergubernamental del 20 de mayo de 1996, el cual estableció una serie de programas de trabajos conjuntos. Dicho acuerdo de cooperación se actualizaba una vez cada dos años, teniendo en cuenta los últimos avances científicos y tecnológicos.

En los últimos cinco años se han desarrollado más de 20 proyectos bilaterales de investigación; entre los más importantes están la creación de microsatélites para monitorizar la superficie de la tierra, proyecto implementado de manera conjunta con la Universidad Estatal M. V. Lomonósov de Moscú, el Instituto Skobeltsyn de Investigación Científica de Física Nuclear y la Universidad Nacional Autónoma de México, así como la elaboración de modelación matemática y experimental de procesos biológicos, proyecto realizado por la Universidad Estatal M. V. Lomonósov de Moscú y la Benemérita Universidad Autónoma de Puebla.

Entre otros ejemplos exitosos de colaboración científica se puede mencionar la formación de nuevos métodos para el diagnóstico y el tratamiento del cáncer, realizada conjuntamente por la sucursal siberiana de la Academia de Ciencias de Rusia y la Universidad Nacional Autónoma de México, y el desarrollo de métodos matemáticos aplicables para el análisis de grandes sistemas computarizados, implementado por el Centro Dorodnitsyn de Computación de la Academia de Ciencias de Rusia y la Universidad Autónoma del Estado de Morelos. Todos los proyectos conjuntos mencionados han llevado a avances reseñables para las disciplinas analizadas.

Debe admitirse que existe un cierto optimismo en la creciente comprensión por parte de las elites políticas rusas de la necesidad de cambiar el centro de gravedad de la interacción económica con México al área de cooperación científica y técnica. Es en el campo de las altas tecnologías donde Rusia es más competitiva. Ambos Estados están tratando de dominar un modelo de desarrollo innovador. En un contexto global en el que los problemas del desarrollo potencial científico y técnico en estas condiciones son de particular relevancia, se ha creado el potencial de complementariedad, especialmente porque el área de nuevas tecnologías es el lado más fuerte de Rusia, y México las necesita con urgencia. En los últimos años se ha prestado mayor atención al fortalecimiento de la cooperación bilateral en el campo de la alta tecnología y la innovación, así como a la realización de investigaciones conjuntas sobre los usos pacíficos del espacio cósmico. En 2013, el satélite comercial de comunicaciones mexicano Satmex 8 se lanzó desde el cosmódromo de Baikonur. Junto con esto, los expertos de la Agencia Espacial Federal de Rusia Roscosmos y la Agencia Espacial Mexicana comenzaron la colaboración para elaborar un acuerdo intergubernamental sobre cooperación en el campo de la investigación y el uso pacífico del espacio ultraterrestre.

Gracias a la firma del acuerdo bilateral sobre los usos pacíficos de la energía nuclear, ratificado en julio de 2015, y la implementación de proyectos científicos y técnicos conjuntos, se ha dado un nuevo impulso al desarrollo de esta área de cooperación. Las relaciones bilaterales en el campo de la ciencia y la tecnología continúan desarrollándose activamente, debido, entre otros aspectos, al hecho de que actualmente en México trabaja un buen número de especialistas rusos, científicos y profesores universitarios, ocupando empleos tanto temporales como fijos, cuya profesionalidad continúa siendo incondicional entre los mexicanos (Farah y Reyes, 2014).

Cabe señalar que, desde mediados de la década de 1990, sigue teniendo lugar un intercambio de estudiantes y científicos sobre la base de acuerdos intergubernamentales. En particular, proporcionaron la asignación de becas anuales para estudiantes y científicos (de cada país hasta quince 
personas) que tuvieron la oportunidad de cursar un programa de estudio o realizar investigaciones científicas durante un periodo de tres meses a un año. Del lado mexicano, junto con las universidades capitalinas, también participan en estos programas las universidades de diferentes estados de la república.

Entre otras cosas, con el apoyo de la Asociación Latinoamericana Rusa, cuya oficina de representación se abrió en la Ciudad de México en 2014, Rusia continuó ofreciendo a los jóvenes mexicanos diversas formas de ingreso a maestrías y doctorados, así como a los cursos de idiomas y escuelas de verano especialmente organizadas, algunos de forma gratuita. Las principales áreas de conocimiento científico, y más populares entre los mexicanos que desean viajar a Rusia para estudiar, son las carreras médicas y técnicas, las líneas de formación de ingeniería del transporte aéreo e ingeniería y tecnología, además de las humanidades, tales como la economía y las relaciones internacionales. En un futuro próximo, se planea concluir un acuerdo bilateral de reconocimiento mutuo de títulos, diplomas y grados académicos de educación superior universitaria. Además, ya ha sido acordada la celebración anual del Foro de Rectores de Rusia y México. A través del programa ya lanzado Educación en Rusia, Moscú planea promover aún más la enseñanza del idioma ruso en México.

En febrero de 2018, la V Reunión de la Comisión Mixta de Cooperación Cultural, Educativa y Deportiva entre México y Rusia se celebró en Moscú, en la cual se firmó un protocolo de cooperación en dichas áreas para el periodo 2018-2020. La delegación incluía altos funcionarios de los ministerios de Asuntos Exteriores, Cultura, Educación y Ciencia de ambos países. Dentro de este evento la atención se centró en el refuerzo de las alianzas a largo plazo entre los Ministerios de Cultura, así como entre los museos mexicanos más famosos, como la Galería Tretiakov y el Museo Pushkin de Moscú (Museo Estatal de Artes Plásticas).

Mientras que la apertura del Centro Ruso de Ciencia y Cultura en la Ciudad de México todavía se discute, en 2013, en la ciudad de Puebla, se abrió la Casa de Cultura México-Rusia, uno de los centros industriales y culturales mexicanos más importantes. Es de destacar que el principal promotor ideológico para la fundación de un nuevo centro cultural ruso en el país azteca fue un mexicano, graduado por la Universidad Rusa de la Amistad de los Pueblos.

En junio de 2015 se celebró el foro Días Mexicanos en Ekaterinburgo, en cuyo marco se presentó una amplia gama de programas dedicados al arte, la cultura, la gastronomía, la economía y el turismo de México. La región de Sverdlovsk ha comenzado a suscitar un interés particular de los empresarios mexicanos, confirmado con la firma del Acuerdo de Entendimiento para promover y fortalecer las relaciones entre México y la región de los Urales.

En cuanto a la región central de la Federación de Rusia, en junio de 2017 se firmó un memorando de cooperación en el campo de la atención médica entre Moscú y la Ciudad de México, que sirve como base para la implementación de una serie de proyectos en el campo de la formación profesional y la organización de la atención médica.

Además, la cooperación en el campo de la cultura se está desarrollando gradualmente. La exposición retrospectiva de Frida Kahlo, que por primera vez tuvo lugar en Rusia, fue caracterizada por la prensa rusa como uno de los eventos culturales más importantes de 2016. 34 obras provenientes del Museo Dolores Olmedo, la Galería Arville y la colección personal del nieto de Diego Rivera, se exhibieron con tremendo éxito en el Museo Faberge en San Petersburgo, generando un interés sin precedentes entre los visitantes rusos. La exposición Viva la Vida: Frida Khalo y Diego Rivera, celebrada a principios de 2019 en la sala central de exposiciones Manezh, recibió reseñas excepcionalmente elogiosas de los críticos, así como del público de Moscú. En el marco de esta 
doble retrospectiva se exhibieron las obras más conocidas de Frida Khalo, además de sus vestidos favoritos, que juntos lograron crear para los visitantes una sensación de inmersión completa en el mundo de emociones y sufrimientos internos de la artista mexicana.

Las relaciones entre México y Rusia se intensificaron notablemente después de que en 2016 Estados Unidos comenzó a amenazar con militarizar aún más la zona de la frontera entre México y Estados Unidos, y también con suspender el Tratado de Libre Comercio de América del Norte, empujando deliberadamente al gobierno mexicano a una búsqueda de nuevos socios comerciales globales (Mora, 2019). Al mismo tiempo, tales acciones de la administración estadunidense han causado un incremento del sentimiento antiestadunidense en la sociedad mexicana. Esto se reflejó en el hecho de que la victoria en las elecciones presidenciales de 2018 por primera vez en la historia de México la alcanzó un representante del partido de izquierda Movimiento de Regeneración Nacional (Morena), Andrés Manuel López Obrador, quien declaró repetidamente que en caso de ganar el poder opondría una digna resistencia al gobierno de Estados Unidos, además de desarrollar otras direcciones de su política exterior, incluyendo Rusia. Al mismo tiempo, el hecho de una creciente "amistad" ruso-mexicana inesperada comenzó a ser muy preocupante para Washington.

No obstante, la administración de López Obrador aún no ha establecido un concepto claro de política exterior propia a seguir en el futuro cercano. El escenario más probable, considerado por Moscú, es que, en cuanto a las relaciones internacionales, México va a improvisar, ajustándose a la situación política actual. Y esto significa que su política exterior ya no será consistente, sino que gradualmente se construirá sobre improvisaciones, cada vez adaptándose a un momento definitivo de la coyuntura. Y, entre otras cosas, esto se reflejará en las relaciones ruso-mexicanas, las cuales bajo las tres administraciones anteriores se iban desarrollando de manera bastante secuencial a pesar de la preponderancia absoluta de la cooperación con Estados Unidos en la agenda exterior mexicana. Y, teniendo en cuenta el hecho de que la nueva administración mexicana dio prioridad absoluta precisamente a la solución de los problemas internos del país, se puede predecir que, en el marco de su política exterior, se asignará un papel especial a la diplomacia económica. Lo que abre nuevas perspectivas para el desarrollo de las relaciones comerciales ruso-mexicanas a un ritmo más rápido (Jeifets, 2015).

\section{Conclusiones}

A la vista de lo analizado, se hace patente que Rusia y México tienen muchos puntos de contacto en la política global. El rango de interacción se está expandiendo y fortaleciendo, aunque a un ritmo bastante lento. Se pueden identificar varios obstáculos para un mayor desarrollo de las relaciones entre los dos países. El primero sucede porque, históricamente, Moscú siempre ha sido bastante cauteloso en sus relaciones con México y nunca ha tratado de aumentar significativamente su presencia en la esfera de la influencia natural de Washington. La Rusia moderna basa la mayor parte de su interacción comercial con América Latina en la esfera militar, pero México, en este sentido, presenta pocas perspectivas debido a sus estrechos vínculos con Estados Unidos, desde donde se importa la mayor parte del equipo militar.

El gobierno mexicano está mucho menos orientado ideológicamente que los regímenes izquierdistas de América Latina y, por razones objetivas, no difunde el antiamericanismo. Una barrera importante es la incorrecta estrategia geopolítica y comercial de Moscú hacia América Latina. Teniendo en cuenta el alto grado de integración de la economía mexicana con su vecino del norte (80\% de las exportaciones mexicanas depende de Estados Unidos), la dependencia de México de 
Estados Unidos es el factor limitante clave en el desarrollo de las relaciones ruso-mexicanas. En este sentido, la parte rusa aún no puede abandonar por completo los estereotipos bastante estables de la percepción de México como el "patio trasero" de Estados Unidos, donde "no hay nada que buscar".

A pesar de todas sus aspiraciones, a finales de la segunda década del siglo xxi las elites gobernantes mexicanas no podrían realmente intensificar notablemente la cooperación con Rusia. Por paradójico que parezca, la llegada de Donald Trump a la Casa Blanca lo hizo por ellos, pues dio el impulso necesario a las acciones de acuerdo con la estrategia "el enemigo de tu enemigo es mi amigo". Se espera que el nuevo presidente de México, Andrés Manuel López Obrador, desarrolle con mayor éxito el curso de las relaciones hacia un acercamiento con el Kremlin. Remarcable es que López Obrador aceptó la invitación para hacer una visita oficial a Moscú.

Incluso permaneciendo en un orden mundial cambiante, la cooperación tradicional entre Rusia y México requiere desesperadamente nuevos impulsos y contenidos, dado el tamaño y las capacidades de Rusia y México. Para intensificar el diálogo ruso-mexicano, es importante asegurar y mantener una cooperación más constructiva al más alto nivel estatal y también fortificar los enlaces interparlamentarios, involucrando más ampliamente una vasta gama de comités especializados en asuntos internacionales. En el campo de la cooperación comercial y económica, parece aconsejable tomar una serie de medidas que podrían intensificarla significativamente, tales como el establecimiento del Consejo Empresarial Ruso-Mexicano de Comercio, la inversión, la cooperación económica y tecnológica, la fundación de la oficina de representación de la Cámara de Comercio e Industria de Rusia en México, la disminución de derechos de aduana y la introducción de beneficios adicionales para los exportadores.

El enfoque pragmático de ambos departamentos de economía extranjera, organizaciones empresariales, administraciones regionales, es decir, todas esas estructuras, que ayudan a las empresas mexicanas a promover productos y servicios competitivos en el mercado ruso, permanecerán como el factor determinante para las futuras relaciones comerciales y económicas de México y Rusia, independientemente de los detalles de la política económica exterior del nuevo presidente mexicano. En la etapa actual, las funciones de dispensación de una amplia gama de servicios especializados en comercio internacional y aduanas cumplen la Asociación Nacional de Importadores y Exportadores de la República Mexicana y la Embajada de Mexico en Rusia. En 2007 se creó el Consejo Coordinador de Compatriotas Rusos en México, que está oficialmente registrado como organización no gubernamental. Se ha convertido en una base importante para proteger los intereses y derechos de la diáspora rusa, así como en un instrumento para aumentar el prestigio de nuestro país en la sociedad mexicana. En 2019 se reanudó el trabajo del Comité de Empresarios Rusia-México en la Cámara de Comercio e Industria de la Federación de Rusia. También vale la pena señalar que la Comisión bilateral ruso-mexicana de consultas sobre cuestiones multilaterales surge como un instrumento para ampliar los contactos entre los sectores empresariales de ambos países, con el fin de fortalecer los vínculos comerciales, usando mecanismos de reuniones regulares.

El final de la segunda década del siglo xxi se caracteriza por la aparición de las condiciones favorables, manifestadas en las aspiraciones de México de diversificar sus relaciones externas, permitiendo predecir la posibilidad de una renovación radical de las relaciones ruso-mexicanas, lo que nos dará la posibilidad de adaptarse a las nuevas realidades en el futuro cercano y corresponder al potencial de nuestros países. Se espera que en la agenda de política exterior del nuevo presidente mexicano, Rusia ocupará un lugar que corresponde a su potencial geopolítico, y las relaciones ruso- 
mexicanas comenzarán a desarrollarse a un ritmo más rápido en comparación con otros Estados latinoamericanos. Respecto a Rusia, este país necesita reconsiderar los estereotipos establecidos de percibir a México como un Estado-conductor de la política de Washington, y transferirlo de manera gradual al rango de socios prometedores. Es previsible que la nueva alineación geopolítica de las fuerzas en el contexto internacional finalmente permita romper el paradigma de las relaciones ruso-mexicanas, que se ha arraigado desde finales del siglo $\mathrm{xx}$. Y sus propias casuísticas, como la baja prioridad y el simbolismo, caerán en el olvido para siempre.

\section{LISTA DE REFERENCIAS}

Borovkov, A. N. (1999). Mexico is at a new turn in economic and political development. Moscú: ILA RAS. Borovkov, A. N., Bulavin, V. I., Glinkin, A. N., Sheremetyev, I. K., Vizgunova, Y. I., Kuzmin, V. V., Teperman, V. A. (2005). Mexico in the modern world and in the system of international relations of the Russian Federation. Moscú: ILA RAs.

Cárdenas, H. (1993). Historia de las relaciones entre México y Rusia. México: Secretaría de Relaciones Exteriores/Fondo de Cultura Económica.

Davydov, V. M. (2003). The effect of adaptation reform: From Latin America to Russia. Moscú: ILA RAs.

Davydov, V. M. (2016). Determination of the development of Latin Caribbean America. The combination of global and regional issues. Moscú: ILA RAS.

Davydov, V. M. y Kalashnikov, N. V. (eds.) (2006). Russia and the Ibero-American World in the 21st century: Horizons of development and cooperation. Moscú: ILA RAS.

Farah, D. y Reyes, L. E. (2014). Russia in Latin America: A strategic analysis. PRISM. The Journal of Complex Operations, 5(4), 101-113.

Federal State Statistic Service (2020). Official statistics reports. External economic activities. Russia. 2020. Rusia: Federal State Statistic Service.

Garza, H. (1988). Las relaciones entre México-URSS. Foro Internacional, 28(4), 760-769.

Garza, H., y Chacón, S. (eds.) (2002). Entre la globalización y la dependencia: la política exterior de México, 1994-2000. México: El Colegio de México/Tecnológico de Monterrey.

Jeifets, V. (2015). Russia is coming back to Latin America: Perspectives and obstacles. En A. Serbin, L. Martínez y H. Ramanzini, Jr. (eds.), Anuario de la Integración Regional de América Latina y el Caribe-América Latina y el Caribe y el nuevo sistema internacional: Miradas desde el Sur (núm. 11, pp. 90-121). Buenos Aires: CRIES.

Kosevich, E. Y. (2017). Mexico in search of the UN security council reform. Actual Problems of Russian Law, 12, 148-159. DoI: 10.17803/1994-1471.2017.85.12.148-159

Kosevich, E. Y. (2020). Mexico in the system of geopolitical coordinates of the beginning of the 21st century. Moscú: Nestor-Historia.

Kuteinikov, A. E. (2012). International intergovernmental organizations: Theoretical and sociological analysis. San Petersburgo: Universidad de San Petersburgo.

Latin America in modern international relations (2009). Moscú: Universidad Estatal de Moscú de Relaciones Internacionales del Ministerio de Asuntos Exteriores de Rusia.

Lavut, A. A. (2012). Pacific alliance of the Latin American four. Latin America [Latinskaya Amerika], $4,11-24$.

Martínez, C. B. (2010). Interés nacional y equilibrio de poder en las relaciones entre Rusia y México de 1890 a 2010. Revista Mexicana de Política Exterior, 91, 108-116. 
Martínez, C. B. y Garza, H. (2013). La geopolítica de México y sus efectos en la seguridad nacional, 1820-2012. Foro Internacional, 53(1), 57-106.

Martynov, B. F. (ed.) (2014). Organized crime is a security challenges in Latin America. Moscú: ILA RAS.

Martynov, B. F. (ed.) (2017). Modern organized crime in Latin America and the Caribbean. Moscú: Ves Mir Publishing House.

Mexico in the modern world and in the system of international relations of the Russian Federation. (2005). Moscú: ILA RAS.

Mora, L. M. (2019). La política comercial de México durante el gobierno del presidente Enrique Peña Nieto (2012-2018). Foro Internacional, 59(3-4), 958-987.

Ortega, M. y Sizonenko, A. (1990). México y Rusia en la primera mitad del siglo XIX. Ciudad de México: Archivo Histórico Diplomático Mexicano-Secretaría de Relaciones Exteriores.

Patiño, C. A. (2014). EEUU-Rusia: chacia una reconfiguración geopolítica de América Latina y el gran Caribe? Análisis Político, 82, 196-211.

Patrick, S. (2014). Uncontrollable world. Arguments in favor of "good enough" global governance. Russia in the Global World, 1, 8-27.

Picarella, L. y Scocozza, C. (2018). Populismos y populistas: las evoluciones de un paradigma entre México y Rusia. Revista Humanidades, 9(1), 1-30. Dor: 10.15517/h.v9i1.35286

Primakov, E. M. (2014). Challenges and alternatives to the multipolar world: The role of Russia. Moscú: Universidad Estatal de Moscú.

Rogozhin, A. A. (2016). Trans-Pacific partnership-expansion potential. En V. G. Khoros y D. B. Malysheva (eds.), West-East-Russia 2015. Yearbook. Moscú: Iмeмо.

Santos, G. M. (2010). Rusia en América Latina (Servicio de Investigación y Análisis No. SPE-ISS06-10). Ciudad de México: Centro de Documentación, Información y Análisis, Cámara de Diputados. Retrieved from Centro de Documentación, Información y Análisis, Cámara de Diputados. Recuperado de http://www.diputados.gob.mx/sedia/sia/spe/SPE-ISS-06-10.pdf

Sheremetyev, I. K. y Lapshev, E. G. (1990). Mexico: Capitalism and society. Moscú: Academia de Ciencias de Rusia.

Shkolyar, N. (2019). Mexico opens up new opportunities for Russian business. Latinskaia Amerika, 10, 22-30. DOI: 10.31857/S0044748X0006408-7

Shulgovsky, A. F. (1967). Mexico at a sharp turn in its history. Moscú: Education.

Simonova, L. N. (ed.) (2013). Latin America on the path of economic modernization. Moscú: IMEMO RAS.

Sizonenko, A. (2004). Latin America and Russia. International Affairs, 50(2), 52-60.

Suárez, A. V. (2015). La industria de la defensa y seguridad en America Latina. Madrid: IDs.

USA and Latin America after the end of the cold war (2004). Moscú: Science.

Zaemsky, V. F. (2008). Recent history of United Nations organization reform. Moscú: MGIMo. 